\title{
Sobre la racionalidad de la esfera afectiva y su vínculo con la razón teórica en la ética de E. Husserl
}

\section{(On the rationality of the affective sphere and its relationship with theoretical reason in E. Husserls's ethics)}

\author{
Celia CABrera
}

Recibido: 11 de enero de 2013

Aceptado: 22 de mayo de 2013

\section{Resumen}

El objetivo del artículo es evaluar en qué medida la primera tematización de Husserl de la conciencia afectiva logra extender el concepto de "razón" a esta esfera y determinar si el modo en que lo hace amenaza su autonomía respecto de la razón teórica. Nuestro eje será el problema de la constitución originaria del valor en los actos no objetivantes del sentimiento, tema anunciado en las Investigaciones Lógicas que adquiere un rol central en las primeras reflexiones éticas de Husserl.

Palabras clave: Husserl, razón, afectividad, valor, ética.

\begin{abstract}
The aim of the article is to evaluate to what point does Husserl's first insight into affective consciousness manage to extend the concept of "reason" to this sphere and analyses whether the way this is accomplished threatens its autonomy regarding theoretical reason. Our main core will be the problem concerning originary value constitution in not objectifying acts of feeling, topic already announced in the Logical Investigations, that plays a central role in Husserl's early reflections on ethics.
\end{abstract}

Keywords: Husserl, reason, affectivity, value, ethics. 


\section{Introducción}

Desde muy temprano, especialmente en sus años de Göttingen, Husserl dedicó gran parte de sus manuscritos a la descripción de una esfera que ha sido soslayada por sus investigaciones sobre la conciencia lógico-cognitiva: las vivencias afectivas. Como señala Ullrich Melle, editor de las Lecciones sobre ética y teoría del valor de 1908-1914, las primeras reflexiones de Husserl sobre la conciencia emocional y volitiva han constituido un esfuerzo por incluir la esfera axiológico-práctica en una teoría y crítica fenomenológica de la razón comprensiva y unificada1. Este interés se relaciona con la idea de que la razón no es algo que sólo pertenezca a la esfera intelectual sino que rige toda la vida humana. Esta vida no sólo consta de actos teóricos sino también de actos emotivos y volitivos de modo que también ellos deben ser comprendidos bajo normas de la razón. Pero la investigación de la dimensión afectiva no sólo respondió en la filosofía de Husserl a un interés epistémico, sino también a la intención de elaborar una ética que, sin renunciar a la racionalidad, tome a la vida sentimental como su fundamento.

En términos generales, pueden distinguirse tres etapas en el pensamiento ético de Husserl. Un periodo temprano en el que, fuertemente marcado por Brentano, predomina su interés por hallar en la esfera emocional y volitiva leyes análogas a las del pensamiento lógico; un periodo de transición, donde, aunque con nuevos matices, subsisten aún algunos tópicos del primer periodo ligados al punto de partida racionalista formal y una fase tardía en la que, ya bajo la influencia de Fichte y de la ética material de Scheler, Husserl realiza un giro hacia una ética personalista fundada en los valores del amor. Nuestro trabajo se centrará en el primer periodo, es decir, en el momento en que comienza a configurarse la ética como un campo propio en la fenomenología. Este periodo corresponde a los tres cursos dictados en la Universidad de Göttingen entre 1908-1914, editados bajo el título Vorlesungen über Ethik und Wertlehre 2 en el tomo XXVIII de la serie Husserliana ${ }^{3}$.

El primer interés ético de Husserl ha sido, al igual que en Brentano, la refutación del escepticismo que subyace a toda ética relativista. Frente al peligro de las posiciones que anulan la problemática ética se impone ante todo, para Husserl, la tarea de fundamentar científicamente la ética i.e., de modo que haga posible a los hombres un orden racional del vivir y el hacer en vistas a los fines superiores de la

\footnotetext{
1 Cf. Melle (2002), p. 232.

2 En adelante: VEW

3 El periodo de transición, comprende las lecciones dictadas en Freiburg en 1920-1924, editadas bajo el título Einleitung in die Ethik -Husserliana XXXVII (Aunque el giro en la concepción ética de Husserl ya se encuentra anunciado en las "Tres conferencias sobre el ideal de humanidad en Fichte" Husserliana XXV). El periodo tardío comienza con los artículos sobre Renovación, preparados entre 1922-1924 para la revista The Kaizo -Husserliana XXVII.
} 
vida. Retomando la concepción de las disciplinas aplicadas presentada algunos años antes en los Prolegómenos a la lógica pura Husserl considera que el fundamento esencial de la ética como disciplina práctica normativa debe provenir de una ética pura $^{4}$. De este modo, el esfuerzo en esta época consistirá en fundar la ética de modo puramente formal. Conforme a la convicción de que la valoración y la voluntad están gobernadas por leyes a priori de carácter ideal que no pueden ser reconducidas a ningún elemento empírico, dicha fundamentación se realizará siguiendo el modelo de las Investigaciones Lógicas: mediante la refutación del psicologismo y a través de la exposición de las leyes a priori de la axiología y la práctica. Como adelantamos, atendiendo a esta exigencia, las Lecciones de 1908-1914 se proponen como tema principal el establecimiento de un fuerte paralelismo entre la lógica, en tanto ciencia de las leyes del pensamiento correcto, y la ética, en tanto ciencia de las leyes del correcto querer y actuar. La idea aquí es que, así como a la lógica formal corresponde una teoría del conocimiento formal, sucede algo análogo con la axiología y la práctica formal respecto de las disciplinas fenomenológicas inherentes por principio a ellas, esto es, la teoría de la valoración y la teoría de la voluntad. ${ }^{5}$ Ahora bien, el establecimiento de este paralelismo debe ser legitimado, por un lado, mediante la comprensión de sus distintos dominios bajo un concepto genérico de razón y, por otro, evitar la posibilidad de concluir que tal paralelismo no haría más que ocultar que sólo hay una única razón, la razón lógica, y que las otras especies de razón sólo serían su aplicación en distintos ámbitos. En tanto "la soberanía universal (Allherrschaft) de la razón lógica es innegable", el problema sería que ella llegue al punto de subsumir la esfera axiológico-práctica bajo sus propios principios. Una exploración de la razón en general es naturalmente una operación lógica, dice Husserl, ¿Pero esto significa que toda razón es ella misma una razón lógi-

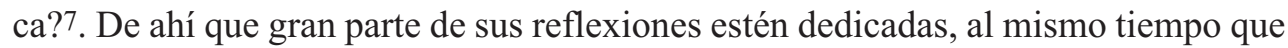
al desvelamiento de su vínculo, a la demarcación esencial de la diferencia específica entre razón teórica y razón axiológico-práctica.

Considerando que el paralelismo entre los distintos "lados"8 de la razón se basa en el paralelismo entre tipos de actos y que ellos remiten a dominios específicos de objetos, el problema se extiende a los tres niveles: Para dar cuenta de la autonomía de la razón axiológico-práctica Husserl debe primeramente lograr distinguir los

\footnotetext{
${ }^{4}$ Cf. VEW, p.13. En los Prolegómenos Husserl había sostenido en relación a la dimensión aplicada de la lógica que todo cuanto hay de científico en las ciencias práctico-normativas coincide con lo que hay en ellas de teorético puro. (Cf. Husserl, 2009, 197)

5 Husserl, (1988), p. 4.

${ }^{6}$ Ibidem, p. 59. Traducción propia. En adelante, en todos los casos en los que no se especifique traducción, la traducción al español es propia.

7 Ibidem.

8 Para evitar la idea de que la razón se halla dividida en partes separadas, Husserl se refiere a distintos "lados" (Seiten) que constituyen una única razón (Cf. VEW, 228).
} 
actos no objetivantes de esta esfera de los actos objetivantes teóricos y a sus correlatos propios de los objetos teóricos. Y lo mismo vale en sentido inverso, la posibilidad de dar cuenta de la racionalidad de los actos afectivos debe garantizar, más allá de las divergencias, algún rasgo que permita una comunidad entre ellos y los actos teóricos. Esto es, para garantizar la racionalidad de los actos afectivos Husserl necesitará un concepto de razón en el que puedan ser también incluidos los actos no objetivantes.

En virtud de que todo acto del querer se funda en un acto del valorar, en esta época Husserl considera que la base de la ética debe ser una axiología o teoría formal del valor. De este modo, se encuentra en primer lugar ante la difícil tarea de dar cuenta del carácter específico de los actos valorativos y sus correlatos. Pero el intento de captar estos actos en sus determinaciones esenciales y en sus conexiones con otros tipos de actos, es un tema que enfrenta a Husserl a muchas dificultades, dificultades que lo conducen incluso a afirmar que los actos de valoración cumplen una "enigmática función"9. Con todo, la determinación del tipo de correlato que constituyen estos actos y la posibilidad de distinguirlos de los actos teóricos son cuestiones esenciales que se encuentran a la base de la posibilidad de garantizar la racionalidad de la esfera axiológica y de distinguirla de la modalidad lógico-cognitiva. En resumen, el problema al que se enfrenta Husserl en este nivel es esclarecer la naturaleza de los actos de valoración y, correlativamente, la donación del valor de un modo que garantice su status racional evitando, tanto la reducción de la razón afectiva a la razón teórica, como la caída en una posición subjetivista, especialmente el psicologismo, que ya se había ocupado de refutar en las Investigaciones Lógicas respecto de la razón cognoscitiva. Tal es una de las difíciles tareas emprendidas por Husserl en VEW 10 .

El objetivo de este trabajo es, en primer lugar, evaluar en qué medida la primera tematización de Husserl de los actos del sentimiento logra garantizar la racionalidad de la esfera afectiva y, en segundo lugar, determinar si el modo en que lo hace atenta contra su distinción respecto de la esfera lógico-cognitiva. En tanto nuestro análisis se focalizará exclusivamente en el nivel axiológico, que funda la dimensión propiamente práctica, el eje de la reflexión será la problemática husserliana sobre la constitución y donación originaria del valor en los actos no objetivantes afectivos (Gemütsakte) ${ }^{11}$. El análisis se desarrollará en relación a los tres niveles mencionados: especies de razón, tipos de actos y regiones de objetos.

\footnotetext{
9 Ibidem, p. 284.

10 Aunque este problema adquiere un rol central en VEW, los dos libros de Ideas (Husserl, 1985; 2005) presentan importantes reflexiones sobre la esfera afectiva a los que también nos referiremos en el transcurso del trabajo.

11 Aquí es necesaria una aclaración terminológica que más adelante será ampliada. El término alemán "Gemüt", tomado en sentido amplio, refiere, para Husserl, a la esfera afectiva que engloba tanto a los
} 
Sin perder de vista las divergencias fundamentales que existen entre las Investigaciones Lógicas y las obras posteriores de Husserl y en virtud de que el proyecto de una crítica fenomenológica de la razón axiológica como parte de una teoría comprensiva de la razón, que ocupará a Husserl en los años posteriores, se halla en estrecha relación con la teoría de los actos de la Quinta y Sexta Investigación Lógica, nos referiremos, en primer lugar, al modo en que los actos del sentimiento son inicialmente abordados por Husserl en esta obra.

\section{Los actos del sentimiento según las Investigaciones Lógicas}

Sabemos que en las Investigaciones Lógicas Husserl toma algunos principios de la filosofía de Brentano. En primer lugar, la esencia intencional de la vida de conciencia. En segundo lugar, en lo que respecta al modelo de fundamentación de los actos, Husserl no cuestiona la jerarquía que ubica a los actos teóricos en la base de la vida de conciencia. No obstante, realiza allí una profunda crítica al principio de la base representativa de Brentano que le permite, al mismo tiempo, rehabilitarlo. En esta rehabilitación crítica tiene un rol central la distinción entre lo que Husserl denomina "actos objetivantes" y "actos no objetivantes". Veamos cómo sucede esto.

Según Brentano, todo fenómeno psíquico o es una representación o tiene representaciones por base. El motivo fundamental por el cual Brentano considera a las representaciones como actos fundantes es que ellas serían actos simples que poseen referencia intencional de manera directa, esto es, sin la mediación de "modos de referencia". Ahora bien, de acuerdo con Husserl, si tomamos esta afirmación, resultaría que la representación constituiría una "excepción chocante"12 ya que, mientras que cualquier otra esencia intencional es una complexión de materia y cualidad13, que sólo abstractamente se puede descomponer en momentos, la esencia intencional de la representación sería meramente su materia. Pero la materia, advierte Husserl, no puede existir aislada, ella sólo puede alcanzar concreción completándose con momentos que están definidos por ley mediante el género supremo "cualidad de acto", de modo tal que si faltase uno de estos dos momentos no habría esencia intencional. En resumen, la crítica está dirigida al motivo por el cual Brentano considera que la representación es el modo básico de intencionalidad; lo que no admite Husserl es que las representaciones sean consideradas actos fundantes por

actos del sentir (Gefühlsakte) como a los actos volitivos (Willensakte) y, en sentido estrecho, refiere sólo a los actos afectivos del sentimiento. En virtud de que nuestro análisis toma como eje los actos valorativos del sentir (wertende Akte), a partir de aquí, nuestro uso del término "afectividad" traduce el sentido estrecho del término "Gemüt" que deja de lado la dimensión volitiva. Vale aclarar que, de todos modos, el problema que nos ocupa se extiende también a los actos volitivos.

12 Husserl (2009), p. 538

13 El equivalente a lo que Brentano denomina "modo de referencia" es en las Investigaciones Lógicas la "cualidad intencional". 
ser actos carentes de complejidad. Considerando que los actos superiores son, por el contrario, complejos de actos, ya que contienen un acto que da referencia y agregan otro que da el modo de la referencia, el contraste entre actos fundantes y actos fundados en el esquema de Brentano sería equivalente al contraste entre lo simple y lo complejo. Naturalmente, en virtud de uno de los rasgos más propios de su comprensión de la vida de conciencia, Husserl no puede sino rechazar esta concepción. Aún reconociendo que hay actos básicos, toda efectuación intencional es, para él, un entramado o uno-en-otro de partes no independientes entre sí.

Luego de objetar esta doctrina, ${ }^{14}$ Husserl rehabilita el principio de la primacía de los actos teóricos reemplazando la función de las representaciones por su teoría de los actos objetivantes. Poniendo por fundamento este concepto, el principio de la base representativa adquiere, para él, un nuevo sentido. Así las cosas, reformulando el principio brentaniano sostiene que "toda vivencia intencional, o es un acto objetivante, o tiene un acto objetivante por "base»"15. De este modo, Husserl deja en pie la relación de dependencia y fundamentación propuesta por Brentano pero poniendo a la base de toda vivencia intencional a los actos objetivantes entendidos como "un extenso género de vivencias intencionales, que comprende todos los actos considerados, ateniéndose al punto de vista de su esencia cualitativa" 16 . Husserl continúa: "La referencia a una objetividad se constituye siempre en la materia. Pero toda materia es -dice nuestra ley- materia de un acto objetivante, y sólo por medio de uno de estos actos puede convertirse en materia de una nueva cualidad de acto fundada en dicho acto"17. En conclusión, sólo los actos objetivantes pueden poseer materia y el género de los actos que no lo sean sólo la poseerán tomándola prestada, por así decirlo, de la perteneciente originariamente a un acto objetivante que está a su base.

Junto a esta clasificación de las vivencias Husserl realiza una nueva modificación al esquema brentaniano, a saber, la reducción de la división tripartita de las vivencias en representaciones, juicios y actos de amor y odio a dos clases: actos del entendimiento (Verstandsakte) y actos afectivos (Gemütsakte). Estos últimos se dividen, a su vez, en actos del sentimiento (Gefühlsakte) y actos de la voluntad (Willensakte). Esta clasificación de los actos y aquella que los distingue en objetivantes y no objetivantes corren paralelas ${ }^{18}$. Bajo el género de los actos objetivantes

\footnotetext{
14 Husserl rechaza esta doctrina mediante una compleja argumentación en la que, por razones de extensión, no podemos detenernos. Para un análisis más detallado de este tema véase Serrano de Haro (1995).

15 Husserl (Op.cit), p. 578. Las cursivas son del autor.

16 Ibidem, p. 570.

17 Ibidem, p. 579.

18 Esta afirmación, aunque cierta, debe ser restringida pues ambas clasificaciones no se recubren totalmente debido a que Husserl ubica a los actos del entendimiento del preguntar (fragen) junto a los actos que no objetivan.
} 
fundantes Husserl comprende tanto a las representaciones como a los juicios y al referirse a los actos no objetivantes tiene fundamentalmente en mente ciertas cualidades como los sentimientos de alegría, tristeza, etc. Llegado este punto podemos establecer que, según este primer modelo de comprensión de los actos del sentimiento, se trata de cualidades abstractas que necesitan una materia intencional que los oriente y al recibir su materia de los actos teóricos que están a su base, simplemente le añaden a estos una nueva cualidad no objetivante, según la estructura acto fundante-acto fundado. Aunque, para ser más específicos, debemos decir que las cualidades del sentimiento dependen fundamentalmente de la cualidad representativa que les da la referencia intencional y subsidiariamente de la materia intencional del acto. Vemos, pues, que si bien Husserl considera inviable la idea de que el "mero representar" posea en sí mismo referencia intencional, continúa entendiendo, al igual que Brentano, que hay cualidades que dotan de referencia a un objeto y sobre ellas se montan nuevas cualidades no objetivantes que no modifican la materia intencional primaria.

Ahora bien, varios intérpretes ${ }^{19}$ han señalado que el intento de dar cuenta de los actos de valoración desde esta concepción, según la cual los actos fundados sólo cumplen el rol de agregar una cualidad a una materia propia de una cualidad de acto heterogénea, es problemático. La peligrosa consecuencia de este planteamiento sería que, al carecer el acto de valoración de un correlato axiológico propio, lo único que expresaría el juicio de valor sería un estado subjetivo. Es decir, la valoración sólo se relacionaría con una referencia subjetiva que no estaría motivada por nada extrínseco a ella, conduciendo, así, a una "teoría subjetivista del valor"20. En resumidas cuentas, si las propiedades de valor sólo pueden ser aprehendidas como determinaciones de la reflexión ${ }^{21}$, los actos de valoración quedan excluidos de la esfera de la razón. Recordemos, a fin de comprender la relevancia de esta consideración, que el sentido husserliano de razón remite siempre a posibilidades de verificación y al "tener algo como evidente" y la evidencia, enseña Husserl, es el aspecto noético de una verdad que se predica de los objetos mismos.

Este problema, vale aclarar, no es ocasionado por la consideración de los sentimientos como actos fundados, posición que Husserl sostendrá aún con posterioridad a las Investigaciones Lógicas, sino por la comprensión de estos últimos como actos carentes de cualquier tipo de correlato propio, un punto que es innegablemente asumido por Husserl al afirmar que la materia total del acto objetivante fundante es, a la vez, la materia total del acto fundado que lo contiene 22 . Lo que equivale a decir que los actos de valoración no desempeñan ningún rol en la constitución del

\footnotetext{
19 Entre otros Serrano de Haro (1995), p. 72 y Melle (1990), p. 41.

20 Cf. Serrano de Haro (Ibidem).

21 Cf. Husserl (2009), pp. 759-760.

22 Cf. Husserl (Op.cit), p. 578.
} 
objeto, o de modo más claro, que el acto fundante alberga en sí mismo la materia total de lo que puede ser intencionado. Según esto, a fin de evitar esta conclusión, sería necesario dar cuenta de una intencionalidad valorativa que tenga un correlato axiológico propio, aunque esto sólo sea posible en este esquema contando a los actos valorativos entre los actos de género objetivante 23 .

Esta importante observación revela la problemática relación entre los actos de valoración y el tema de la evidencia y la verdad que ocupará Husserl en los años posteriores. Por este motivo es necesario realizar algunas precisiones que, si bien no restan importancia al problema planteado, contribuyen a una comprensión más profunda de su complejidad: Aunque Husserl caracterice a los actos fundados como actos que en sí mismos carecen de materia, dado que ellos están fundados en otros actos que les proporcionan la representación de la objetividad a la que cualifican, no debe pasarse por alto que nunca sucede que carezcan efectivamente de materia. En efecto, resulta evidente que nunca nos sentimos alegres o tristes si no es por algo. En otras palabras, es innegable que el sentimiento respecto de un objeto dado es un momento lógicamente distinto de la experiencia meramente cognitiva del objeto, pero es asimismo innegable que tal momento no puede separarse de la experiencia fundante. Esto es, en la medida en que siempre hay razones objetivas sobre las cuales tal estado se funda, no podemos afirmar que él se reduzca sin más a un momento subjetivo puramente inmanente. Este problema remite, en última instancia, a la posibilidad de adscribir referencia intencional a los actos valorativos. A propósito de este tema, contra quienes conciben a los sentimientos como meros estados, en la "Quinta Investigación" Husserl se propone demostrar su carácter intencional señalando el absurdo que significa negar intencionalidad a los sentimientos bajo la consideración de que ellos deben la referencia a su entrelazamiento con las representaciones en las que se fundan. Husserl recuerda que, contra la interpretación que sólo reconoce carácter intencional a la representación, Brentano ya demostró que se trata aquí de dos intenciones edificadas una sobre la otra: la que proporciona el objeto representado y aquella que proporciona el objeto sentido. En este punto, sostiene enfáticamente que el reconocimiento de que las intenciones secundarias deben su intencionalidad sólo a estar fundadas en las primarias significa al mismo tiempo que ellas tienen también aquello que deben a estas ${ }^{24}$. Lejos de privarlas de referencia intencional, su carácter fundado explica y da cuenta de tal referencia 25 .

\footnotetext{
23 En este sentido, Serrano de Haro considera que "las Investigaciones Lógicas sólo escaparían a esta indeseada consecuencia en caso de poder considerar las propiedades de valor como términos intencionales de actos objetivantes, es decir, si predicados de valor formaran parte de materias intencionales primitivas". (Serrano de Haro, 1995, p. 72). Curiosamente, un pasaje de la "Quinta Investigación" apunta en esta dirección al poner de relieve una propiedad objetiva que opera como fundamento del volverse hacia el objeto. (Cf. Husserl, Op.cit, p. 510).

24 Ibidem, p. 507.

25 Husserl reconoce que hay también sentimientos no intencionales: las sensaciones afectivas (Gefühlsempfindungen) que se distinguen de los actos afectivos y que están fundados en las sensaciones de los sentidos (Sinnesempfindungen).
} 
Aclarado esto, el problema, sin embargo, subsiste. A pesar de que Husserl reconozca carácter intencional a los actos fundados, la verdad en la esfera de estos actos queda desligada en las Investigaciones Lógicas de una posibilidad de legitimación que tenga el carácter de una síntesis de cumplimiento (Erfüllungsynthese) propia y es, por el contrario, relacionada a una percepción interior de la vivencia. Más específicamente, el cumplimiento en los actos fundados es de tal modo dependiente del cumplimiento en los actos objetivantes que es imposible hablar en sentido estricto de una validez propia del sentir. Quizás el caso más ejemplificador de esta posición sea la sección final de la "Sexta Investigación Lógica" donde Husserl afirma sin rodeos que las expresiones de los actos no objetivantes no son más que casos particulares y accidentales de los enunciados de los actos objetivantes 26 .

Muy distinto será el panorama en los años posteriores, donde la búsqueda de una legitimidad que pertenezca intrínsecamente a la vida afectiva será el eje central de los esfuerzos de Husserl. Pero, en virtud de que este objetivo deberá alcanzarse resguardando la diferencia esencial entre actos teóricos y afectivos, la posibilidad de resolver este problema considerando a los actos de valoración como actos objetivantes será, a todas luces, inviable. No obstante, para que los actos del sentimiento puedan ser sometidos a interrogación racional Husserl deberá resolver la aporía a la que lo enfrenta la necesidad dar cuenta de una intencionalidad valorativa susceptible de un cumplimiento propio y de un correlato propio 27.

Ya desde el punto de vista fenomenológico trascendental, Husserl ofrecerá un nuevo modelo de fundamentación que, aunque no renuncia a la primacía de los actos teóricos, amplía el análisis presentado hasta aquí intentado desvelar una peculiar colaboración entre razón teórica y afectiva en la constitución de la objetividad axiológica. A partir de este momento, el interés gnoseológico estará estrechamente vinculado a la problemática propiamente ética: de la posibilidad de dar cuenta del rol constitutivo de los actos no objetivantes, dependerá la posibilidad de establecer una normatividad propia de la esfera axiológica que garantice el paralelismo con la esfera lógica fundamental en la refutación del escepticismo ético. En lo que sigue nos referiremos al modo en que a partir de 1908 Husserl aborda estos problemas que ya habían sido anunciados un tiempo antes en las Investigaciones Lógicas.

\footnotetext{
26 Cf. Husserl, (Op.cit), p. 760.

27 En VEW Husserl expresa su insatisfacción respecto del abordaje de este tema en las Investigaciones Lógicas: “...no pude con los actos afectivos ni con la esencia de la fundación y su relación con los actos objetivantes, como lo muestra la ardua discusión sobre los enunciados de deseo y otros enunciados de ese tipo. Claramente, queda un problema por resolver”. Husserl (1988), p. 337.
} 


\section{El problema de los actos del sentimiento en el marco de la "teoría del valor" (1908-1914)}

Atento a las dificultades hasta aquí expuestas, en VEW Husserl se propone garantizar la posibilidad de una verdadera ciencia axiológica. Con este fin, como hemos adelantado, propone un método de analogía que debe permitirnos hablar de algún tipo de evidencia perteneciente a los actos no objetivantes análoga a la evidencia de los actos cognitivos. Esto es, debemos poder también aquí hablar de intención y cumplimiento. Cabe preguntarse entonces: ¿En qué consiste la donación intencional del valor?

La posición de Husserl en VEW presenta dos vertientes. Por un lado, reafirma el punto de partida anterior según el cual los actos del sentimiento no objetivan en absoluto. Por otro lado, señala que los actos teóricos captan objetos libres de valor, i. e., sólo captan objetos bajo la forma del "ser así". En otras palabras, los actos de la razón teórica se agrupan bajo los actos del intelecto puro que se refieren a objetos exclusivamente de modo no valorativo y no es posible deducir a partir de ellos ningún predicado que no sea estrictamente teorético ${ }^{28}$. En síntesis y en las inequívocas palabras de Husserl: "la teoría no conduce a valores" 29 . Nos detendremos en este último punto ya que incorpora un nuevo matiz a lo que hemos presentado hasta este momento.

La reflexión de Husserl pone de relieve un aspecto fundamental para nuestro análisis, a saber, que la naturaleza teórica del acto fundante es incapaz de explicar el surgimiento del acto de valoración. ¿Qué significa esto? Que la materia de la que surge el acto debe ser afín a la naturaleza no teórica del acto ${ }^{30}$. Lo fundamental es que Husserl reconoce aquí que el valor constituye un contenido ajeno a la razón teórica y con esto que la materia del acto intencional de valoración debe ser ella misma axiológica. Luego, los actos de valoración no pueden reducirse a actos objetivantes no solamente porque la distinción de un dominio específicamente axiológico se vería amenazado, sino debido a que los actos teóricos no podrían en ningún caso acceder a estas propiedades de valor que sólo son originariamente accesibles a la esfera afectiva. De este modo, advertimos que si bien en este contexto Husserl no renuncia a la concepción de los actos valorativos como actos fundados, esto no tiene aquí por fundamento, como en las Investigaciones Lógicas, la ausencia de una materia intencional propia para estos actos, sino que se funda en la diferencia esencial entre actos que captan objetos y actos que se mueven hacia ellos en la estimación pero son incapaces de captarlos. Esta modificación en la concepción de la conciencia emocional anuncia un punto de vista que será también incorporado en la

\footnotetext{
28 Ibidem, pp. 249; 283; 368.

${ }^{29}$ Husserl, (Op.cit), p. 268.

30 Cf. Vicuña Zauschkevich (2010), p. 62.
} 
obra inaugural de la fenomenología trascendental, Ideas I. La explicitación de una intencionalidad valorativa que, a diferencia de aquella que se dirige a "meras cosas" (blosse Sache), revela dimensiones no-cognitivas de sentido del objeto, junto a la extensión de la noción de "conciencia posicional" (tema del que nos ocuparemos en 4.2) desarrolladas por Husserl en este periodo, constituyen uno de los avances más importantes en su indagación de la vida de conciencia. La superación del esquema de las Investigaciones Lógicas salta a la vista: la valoración ya no corre el riesgo de disolverse en una mera referencia subjetiva sino que se asocia a una verdadera intencionalidad afectiva a la que debe corresponder una región noemática propia

Establecido esto se trata, a partir de aquí, de esclarecer en qué consiste la necesaria donación de contenidos axiológicos que son provistos por actos que no pueden objetivar ni explicitar su objeto. Husserl se enfrenta aquí al problema de conciliar el hecho de que sólo los actos intelectivos pueden "poner" objetos con la idea de que los actos de valoración son esenciales a la constitución del valor. En el siguiente pasaje de VEW aparece claramente formulado este problema:

...los actos de la afectividad parecen tener que valer inevitablemente como actos constituyentes para valores, mientras que por otra parte, los valores son objetos y los objetos, como parece igualmente inevitable, solo pueden constituirse en actos de conocimiento (...) De este modo, no comprendemos ni cómo es posible distinguir razón teórica y razón axiológica, ni como los actos valorativos pueden tener una relación esencial con el darse de los valores (Wertgegebenheit) en la medida en que sólo los actos teóricos o actos del conocimiento deben proporcionar objetivación. O más aun: vemos que puede distinguirse razón teórica y razón axiológica pero la distinción se nos vuelve incomprensible cuando buscamos captarla más de cerca. Y nuevamente, vemos que los actos de valoración son esenciales a la constitución de los valores; pero si nos preguntamos cómo pueden funcionar constitutivamente, llegamos a aporías: sólo los actos objetivantes pueden, sin embargo, constituir. ${ }^{31}$

Es esta aparente "función enigmática" (rätselhafte Funktion) la que sustenta la tentación de considerar los valores como predicados psíquicos, "volatilizarlos en la esfera psicológica" 32 . Sin embargo, más allá de las dificultades que representa esto para Husserl y a fin de evitar que el valor se disuelva en la relatividad del valorar, Husserl insiste en la objetividad de las propiedades de valor. Así, en las lecciones de Göttingen todo se jugará en la posibilidad de responder a la pregunta por la donación de contenidos axiológicos a actos que son "ciegos" respecto de su correlato. Como claramente resume Karl Schuhmann, el "Hacerse del objeto sin actos que proporcionen objetos: este es el problema de Husserl" 33 .

\footnotetext{
31 Ibidem, p. 277.

32 Ibidem, p. 284.

33 Schuhmann (1991), p. 108.
} 


\section{Hacia una resolución del problema de la constitución y la donación originaria del valor}

Si nos atenemos al método de analogía propuesto por Husserl, se presentan tres posibilidades: o bien concebimos el acto en el que el valor es dado originariamente como análogo a la percepción sensible, o a una toma de posición, o a un acto categorial. En cada uno de estos casos el valor tendría un status ontológico distinto: en el primer caso sería similar a un objeto sensible, en el segundo sería un carácter noemático y en el tercero un objeto de nivel superior constituido activamente 34 . Ahora bien, la necesidad de mantener la diferencia entre el acto de valoración (Wertung) y el juicio de valor (Werturteil), que implica una objetivación categorial, vuelve inviable la tercera posibilidad. El motivo fundamental reside en que nuestro problema es esclarecer la experiencia en la que el valor es dado originariamente al sentimiento $y$, en virtud de que los juicios no hacen más que articular categorialmente una verdad de la experiencia, un contenido vivido, su verdad está fundada en una verdad más originaria del sentir, que es la que aquí necesitamos desvelar. Analizaremos, entonces, las dos primeras interpretaciones: la consideración de los actos de valoración como actos análogos a la percepción sensible y la idea de que ellos constituyen una particular toma de posición a fin de esclarecer si es posible a partir de ellas ofrecer una explicación que garantice la racionalidad de los actos no objetivantes afectivos.

\subsection{El acto de valoración como percepción del valor (Wertnehmung)}

Atendamos a la primera posibilidad. De acuerdo con ella, un acto análogo a la percepción sensible (Wahrnehmung) provee el material axiológico que constituye el correlato del acto afectivo sobre el que luego se funda el juicio categorial de valor. Así como la percepción sensible consiste en una interpretación de datos sensibles, la percepción del valor (Wertnehmung) consistiría en una interpretación de datos que son, en este caso, sentimientos sensibles fundados en datos sensibles percibidos. Los sentimientos sensibles proporcionarían, de acuerdo con esto, la materia para una apercepción que tiene lugar mediante una noesis específicamente afectiva.

Esta interpretación que permite afirmar que toda conciencia que constituye un objeto de valor pertenece a la esfera de la afectividad, conservando a la vez la diferencia de dichos actos respecto de los actos de la razón teórica, es anunciada en VEW y profundamente desarrollada en el parágrafo 4 de Ideas II, donde Husserl sostiene la posibilidad de concebir actos constituyentes que difieren de los actos de carácter dóxico ${ }^{35}$. Husserl introduce allí una distinción entre actos teóricos y viven-

34 Cf. Melle (2002), p. 234.

35 Sólo en un Apéndice de VEW (Husserl, 1988, p. 362) Husserl se refiere a la apercepción del valor en términos de Wertnehmung. Allí, al igual que en Ideas I sostiene en general la segunda posición a la que nos referiremos en el siguiente apartado. Cf. Husserl (1985), pp. 279-280. 
cias intencionales pre-dadoras muy esclarecedora para el tema que nos ocupa. Según esto, las vivencias afectivas en cuanto vivencias intencionales son también constituyentes, ellas constituyen estratos objetivos, pero hacia los cuales el sujeto no se halla en actitud teórica. Dice Husserl allí:

... estas difíciles relaciones tienen que ser bien comprendidas y atendidas. Tiene uno que caer en la cuenta de que en la peculiaridad de la actitud teórica y de sus actos teóricos (...) reside el que en cierta manera se encuentren de antemano los objetos que por primera vez llegan a ser teóricos. Así pues, pre-teóricamente ya hay objetos constituidos, sólo que no son objetos teóricamente apropiados, mentados en el señalado sentido, y menos aún objetos de actos que los determinen teóricamente 36

Se trata entonces de objetividades pre-dadas que se constituyen en vivencias intencionales que no les imprimen ningún tipo de configuración lógico-categorial. Esta intencionalidad valorativa se revela a su vez como constitutiva de objetividades de nivel superior, análogas a las categoriales de la esfera lógica. Pero para esto tiene que tener lugar un pasaje desde lo que Husserl denomina "actitud disfrutante", en la cual nos abandonamos en la contemplación emotiva, hacia la actitud teórica en la que el objeto se convierte en "objeto doxo-tético", i. e., objeto de una tesis dóxica mediante la conciencia reflexiva sobre el acto emocional. En virtud de que de todo acto no objetivante pueden sacarse objetividades mediante un giro, todo acto es a la vez implícitamente objetivante, es decir, no sólo está fundado sobre actos objetivantes sino que es objetivante respecto de lo nuevo que él mismo aporta. Lo central es que, según esto, la constitución primigenia del valor se ejecuta en el abandono disfrutante pre-teórico aunque, en sentido estricto, el carácter de objeto se obtiene cuando pasamos de la simple intención a la intuición teórica. Con todo, y este es el punto más relevante, tener vivencias intencionales y ejecutar actos no es equivalente a estar en actitud teórica hacia sus objetos.

De manera que si distinguimos la objetividad fundante, los valores como correlatos de actos afectivos y el objeto que surge del pasaje a la actitud teórica, debemos decir que lo que corresponde a la intuición axiológica es algo que sin ser objeto teórico es, con todas las salvedades del caso, una "pre-objetividad". El hecho de que Husserl en ocasiones se refiera a él como un objeto y en otras no lo haga da cuenta de la dificultad a la que se enfrenta al intentar elucidar la peculiaridad de estos correlatos que, sin ser objetividades en sentido propio, tienen un lado implícitamente objetivo.

En resumen, a fin de resguardar la constitución originaria del valor como perteneciente a la razón valorante, es decir, para garantizar que las determinaciones axiológicas (Wertbestimmheiten) permanezcan siendo axiológicas, es preciso distinguir

36 Husserl (2005), p. 36. 
entonces dos sentidos de objetividad: un tipo de objetividad propiamente axiológica y aquella que la razón teórica puede constituir sobre su base. Esto resulta más claro desde el punto de vista noético donde, correlativamente, habrá que distinguir actos que aportan contenidos objetivables (objektivierbar) pero no objetivan y actos objetivantes en sentido estricto. Este punto es de especial importancia si tenemos en cuenta que en algunas ocasiones Husserl se refiere a los actos del sentimiento como fuentes de una región de ser y de una ontología correspondiente $37 y$ a los valores como una esfera especifica de objetos ${ }^{38}$, mientras que en otros momentos insiste en la necesidad de distinguirlos de los objetos. Lejos de constituir una contradicción, esto se explica porque, si bien los valores pueden constituir algún tipo de objetividad, ella en ningún caso puede ser asimilada a la objetividad de los entes de la naturaleza. Claramente, los valores no pueden ser objetos en el mismo sentido en que lo es una cosa.

Según hemos señalado, sobre la base de esta objetivación implícita, la objetivación propiamente dicha requiere la participación de la razón teórica. En la medida en que es una razón que no comprende, no explicita, ni juzga sus contenidos, Husserl se refiere a la razón valorativa como una razón "muda y, en cierto modo, ciega" 39 . Ahora bien, al mismo tiempo que sostiene que los valores no son explicitables por la razón valorativa, como hemos visto, Husserl ha señalado que los valores son ajenos a la razón teórica. Se impone aquí un interrogante esencial: ¿Cómo la razón teórica podría captar y objetivar contenidos meramente vividos en el sentimiento? La idea de que los actos de valoración, aún siendo no objetivantes, constituyen una toma de posición (Stellungnahme) ofrece una respuesta a este problema.

\subsection{El acto de valoración como toma de posición (Stellungnahme)}

Llegado este punto es preciso detenernos en una consideración. Como hemos visto, la afirmación de Husserl según la cual los valores son ajenos a la razón teórica debe tomarse con ciertos matices ya que, si bien pone de relieve que la razón teórica no constituye originariamente el valor, esto no afecta su posibilidad de constituir una objetividad sobre la base de tal constitución primigenia. El punto central es que, así como la razón afectiva no es suficiente para constituir el valor en tanto objeto categorial, tampoco lo es la razón teórica en la medida en que requiere un contenido axiológico dado al que no puede acceder originariamente. El problema atañe ahora, entonces, a la posibilidad de que la razón teórica se vuelva sobre el contenido vivido y lo objetive. En relación a esto, podemos pensar que la anterior exposición sobre la donación del valor en un acto análogo a la percepción tiene la

\footnotetext{
37 Husserl (1985), pp. 283; 354.

38 Cf. Husserl (1988), p. 283.

39 Ibidem, p. 68.
} 
ventaja de fundar la pretensión de validez del juicio en una cualidad axiológica objetiva análoga a las cualidades sensibles y que, a diferencia del planteamiento de las Investigaciones Lógicas, esta versión no sólo garantiza una cualidad sino también una materia propia para los actos del sentimiento, eliminando el riesgo de que el valor sea producido por el sujeto que valora. Pero, por otro lado, podría sostenerse que por sí sólo esto no soluciona definitivamente el problema de cómo a partir del vivir en la entrega disfrutante puede la razón teórica volverse y objetivar. Esto explica que Husserl sostenga asimismo en VEW y en Ideas I que los actos de valoración constituyen una especie peculiar y única de tomas de posición o mención.

De acuerdo con esto, aunque no son tomas de posición dóxicas, ya que estas pertenecen a la clase de los actos objetivantes, en el noema de nivel superior correspondiente a lo valorado encontramos tanto caracteres de posición, del lado noético, como caracteres téticos, desde el aspecto noemático. Esto es, por un lado, lo valorado es un núcleo de sentido rodeado de caracteres como "agradable", "alegre", etc. y por otro, la conciencia es posicional respecto de los nuevos caracteres, i. e., lo valioso puede ser puesto dóxicamente como "efectivamente" o "supuestamente valioso". Lo novedoso es que se trata de actos que, aunque no objetivan, mientan su contenido. Esta idea permite dar cuenta de la posibilidad de que el conocimiento intervenga captando lo valorado, ya que sólo en la medida en que estos actos son tomas de posición puede la razón teórica encontrar algún contenido valioso (Werthaltung) susceptible de objetivación. Así, en palabras de Husserl, "los actos lógicos sólo iluminan y hacen visible lo que está allí" 40 Es decir, la ulterior objetivación es posible porque en la base de los actos axiológicos hay una posición, ellos ponen al objeto como valioso. Esta extensión de la noción de "toma de posición" a la esfera afectiva adquiere una importancia clave cuando atendemos a la necesidad de garantizar una unidad en el concepto de acto que englobe tanto a los actos objetivantes como a los no objetivantes y que, correlativamente, permita comprender los distintos tipos de razón bajo un mismo concepto. A continuación profundizaremos este punto.

\section{Sobre la racionalidad de la esfera afectiva y su distinción respecto de la razón teórica: Hacia la unidad de la noción de acto y la unidad de la razón}

Llegado este momento, la pregunta que nos ocupa es la que nos propusimos como primer objetivo central de este trabajo: esclarecer de qué modo el acto afectivo, sin ser un acto cognitivo, puede resguardar para sí un rasgo que permita comprenderlo bajo un concepto de razón en sentido amplio. El interés por incluir a los

\footnotetext{
40 Husserl (1988), p. 69.
} 
actos no objetivantes afectivos bajo el concepto razón no es, para Husserl, una necesidad exclusivamente ética. Como señalamos al comienzo, las investigaciones sobre la conciencia afectiva tienen el objetivo de elaborar una teoría de la razón comprensiva y, por sobre todo, unificada. Si bien Husserl ha intentado diferenciar una región de objetos y un tipo de actos que correspondan específicamente al modo afectivo de la razón, como ha señalado Julia Iribarne "La razón no puede comprenderse a partir del modelo del todo y la parte; tampoco se la puede concebir como dividida en dos mitades, una práctica y otra teórica"41. Se trata de un tema de importancia capital para la teoría fenomenológica de la razón.

Volviendo a nuestro tema, en este punto debemos detenernos en un aspecto esencial que ha sido señalado por Ullrich Melle. Este intérprete observa que la concepción del acto de valoración simplemente como un acto análogo a la percepción (Sección 4.1) no es suficiente para garantizar su racionalidad. El problema de esta concepción sería que, así como la percepción (Wahrnehmung) no es aún razón teórica en sentido propio, tampoco podría la percepción del valor (Wertnehmung) ser razón axiológica en sentido propio. Melle pone de relieve que, mientras que la percepción del valor pertenece, al igual que la percepción sensible, a la esfera de la receptividad, la cuestión de la racionalidad se remite siempre a la toma de posición activa y espontánea del ego, tal como tiene lugar en los actos del juicio ${ }^{42}$. Esta observación coloca nuevamente en el centro de la discusión aquello que hemos analizado al comienzo de este trabajo (Sección II), a saber, que la posibilidad de garantizar la inclusión de los actos de valoración entre los actos conformes a la razón depende de la posibilidad de encontrar en los actos no objetivantes un cumplimiento (Erfüllung) que posibilite una legitimación (Rechtausweisung) con anterioridad a toda actividad teórica judicativa.

En este sentido, nos hemos referido en la sección anterior a la concepción de los actos de valoración como tomas de posición. Esto ha garantizado la presencia en los actos afectivos de un "estar dirigido" (Gerichtet-Sein) hacia un contenido intencionado. A fin de evaluar la posibilidad de encontrar también en este nivel una "síntesis de cumplimiento" legitimadora debemos elucidar, ante todo, el particular modo de referencia de las tomas de posición afectivas (Gemütsstellungnahmen). Aunque todas las vivencias intencionales están sujetas a la oposición entre intenciones vacías e intenciones plenificadas, a los diferentes tipos de actos corresponden distintos tipos de plenificación. En efecto, un rasgo característico de los actos afectivos es que en ellos la plenificación de la mención no tiene el carácter de una "síntesis de identificación", como ocurre en los actos teóricos. En estos últimos, la intención vacía se identifica con una intención intuitiva en la que es intencionado el mismo objeto con las mismas determinaciones. En tal identificación se confirma y legiti-

41 Iribarne (2007), p. 27.

42 Cf. Melle (2002), p. 234. Sobre este tema véase también Melle (2012). 
ma la intención del acto vacío. Por el contrario, la plenificación propia del acto fundado de valoración no consiste en la identificación de un objeto. Es decir, en el acto fundante anterior a la valoración y luego de ella, i.e., en la síntesis de reconocimiento, tiene lugar una identificación entre lo mentado y su donación intuitiva. Pero el valorar mismo, al no constituir ninguna objetivación, no implica ninguna identificación ${ }^{43}$. Esto se explica por el peculiar modo de referencia de estos actos. Nos referimos fundamentalmente al hecho de que, a diferencia del resto de los actos, en los actos fundados el estar dirigido es algo documentable en el acto mismo. Así, por ejemplo en la alegría, tenemos además de la alegría el "sobre qué" de la referencia en la forma de una representación fundante. En este sentido señala Husserl que "si adscribimos a una alegría la referencia a un objeto (...) es ello una forma de «referencia a» (Beziehung auf) totalmente distinta de aquella que adscribimos a una percepción, un recuerdo o un juicio" 44 . No obstante, y este es el punto que nos interesa, esto no significa que el pasaje a la plenificación no tenga también aquí el carácter de un cumplimiento. Si bien los actos afectivos no se dirigen a objetos, sino a valores, hemos visto que no son simplemente un "estar junto al objeto mismo sintiendo" sino que sobre la apercepción del valor (Wertapperzeption) se fundan actos superiores de la afectividad, una direccionalidad que tiene lugar en el plano de la conciencia afectiva con anterioridad a todo juicio. Esto significa que aquí encontramos un proceso análogo al perceptivo en el que hay un sentir (fühlen) vacío referido a un objeto y luego se efectúa un proceso de clarificación del sentimiento respecto de ese objeto que confirma o no su carácter valioso. Así, la búsqueda de cumplimento inherente a toda mención posibilita englobar a los actos no objetivantes bajo un a priori teleológico-normativo junto a los actos teóricos ${ }^{45}$. En efecto, este es el concepto de razón que Husserl tiene en mente. "Razón" designa precisamente el a priori teleológico que apunta a relaciones de corrección e incorrección 46 . En fin, es el carácter teleológico de los actos no objetivantes lo que justifica su comprensión en un concepto global de acto $47 \mathrm{y}$, por consiguiente, el paralelismo entre

\footnotetext{
43 E. Vicuña Zauschkevich ofrece un claro esquema de la relación entre actos objetivantes y no objetivantes en el surgimiento del valor en tanto predicado en un juicio. Este autor explica que los actos objetivantes cumplen una doble función: una función tética previa a la captación del valor, que "pone" la unidad objetiva y la función epistémica posterior a la captación del valor, que hace del valor una unidad temática. Entre estos dos momentos se halla la captación del valor a la que nos referimos. Tal momento es posterior a la fundación dóxica del acto y anterior a su constatación teórica, en tanto predicado. Cf. Vicuña Zauschkevich, (2010), p. 60.

44 Husserl (1988), p. 336.

45 Ibidem, p. 340.

46 Ibidem, p. 343

47 Este aspecto ha sido señalado por Urbano Ferrer Santos (2011), quien además demuestra como las consideraciones teleológicas van adquiriendo un rol cada vez más central en la ética de Husserl. Cf. Ferrer (1995); (2012).
} 
razón axiológica y razón teórica que permite a Husserl la refutación del escepticismo ético.

Al momento de abordar el segundo objetivo que nos hemos propuesto, a saber, la distinción de la razón afectiva respecto de la razón teórica, no podemos pasar por alto que indudablemente los límites de la analogía entre los tipos de razón vienen dados por la especificidad de las esferas de validación ya que la atestación axiológica se funda en leyes que corresponden a la esfera específicamente axiológica. Sin embargo, este tema constituye un problema un tanto arduo para la axiología temprana de Husserl.

En el transcurso del trabajo hemos planteado algunos problemas que han salido al encuentro en el análisis de Husserl y hemos intentado dar respuesta a algunos de ellos. Así, la posibilidad de que el conocimiento "alumbre" los actos ciegos del valorar ha sido garantizada mediante la consideración de estos actos como tomas de posición y su racionalidad gracias a su búsqueda de cumplimiento y sujeción a conceptos normativos. Sin embargo, una vez comprendida la donación de un contenido axiológico como correlato de un acto que, a pesar de no ser objetivante en sentido propio, lo es de un modo implícito, la pregunta que se impone con más fuerza aún que aquella por la inclusión de esta esfera bajo el concepto de "razón", es la de si es posible entonces, y cómo lo es, distinguir definitivamente estos actos de los cognitivos y la objetividad del valor de la de las cosas que conocemos. Hemos puesto de relieve que se trata de una objetivación implícita que sólo luego puede ser actualizada por actos teóricos. No obstante, aún con estos matices, es indudable que se trata de una objetivación y este es el punto que puede levantar sospechas. Estas sospechas pueden ser reforzadas por la concepción de los actos de valoración como actos que, aunque presentan una posicionalidad propia, traen también consigo de manera implícita una posicionalidad dóxica. Es decir, si bien, como aclara Husserl, mientras vivimos en la conciencia valorante no nos ocupamos activamente del aspecto dóxico, toda vivencia afectiva es en sí caracterizada como valorar, querer, etc. que conjetura, tiene por sospechoso o duda 48 . Y es este carácter dóxico, aunque implícitamente, extensivo a todo acto lo que vuelve comprensible preguntarse si es posible hablar de un aspecto axiológico de la razón realmente distinto del modo teórico.

Ahora bien, si esto fuera así deberíamos concluir que la posición de Husserl no es más que un intelectualismo que pretende subsumir todas las esferas de la vida a actos intelectivos. Pero, a pesar de su interés por elaborar una ciencia ética alejada del relativismo, no ha sido intención de Husserl confinar todas las formas de racionalidad a la racionalidad lógica. En efecto, Husserl compartió con Brentano la convicción de que el sentimiento cumple un papel esencial en la fundamentación de la

48 Cf. Husserl (1985), p. 271. 
ética y que lo relativo a ella no puede reducirse a conocimientos intelectuales. En virtud de su patente esfuerzo por no caer en una posición de este tipo, debe considerarse que se trata de un problema que surge en gran parte en virtud de la peculiaridad de la tarea propuesta, i. e., de las dificultades inherentes al intento de explicar la legalidad de una esfera de la que tenemos evidencia fenomenológica de un modo innegable pero que al ser ciega y muda, requiere actos del pensamiento para expresar su verdad. Sin embargo, resulta necesario desvelar el modo en que algunos supuestos del marco en el que este tema es inicialmente abordado dificultan la comprensión del sentido de los actos de valoración, al punto de volverla una cuestión aporética. Su clarificación no sólo contribuye a una interpretación más profunda del tema estudiado sino también a la comprensión del viraje que tendrá lugar en el pensamiento ético de Husserl con posterioridad a los años de Göttingen.

\section{Conclusiones}

Hemos visto que las mayores dificultades surgen del intento de diferenciar una región de objetos y un tipo particular de actos que correspondan específicamente al modo afectivo de la razón. En este punto el esfuerzo de Husserl se ha concentrado en dar cuenta del fundamental hecho de que, si bien todo puede ser sometido a una objetivación, no todo surge de una aprehensión de tal tipo. En otras palabras, que es posible "estar vuelto" hacia un objeto sin captarlo teóricamente y en consecuencia, la existencia de una región de determinaciones que constituyen correlatos intencionales pero que no son primariamente accesibles a la objetivación teórica. Sin embargo, cuando atendemos al origen de las dificultades que afronta Husserl al momento de distinguir los valores respecto de las representaciones descubrimos algunos rasgos que explican la aporía a la que se ha visto conducido. Nos referimos con esto a una particular interpretación de la relación entre los distintos tipos de actos y del vínculo entre sus correlatos y fundamentalmente a una concepción de la constitución y de la intencionalidad de la que resulta que sólo los actos fundantes pueden constituir49.

No hemos pasado por alto el importante avance que representó para la concepción husserliana de la vida afectiva el desvelamiento de nuevos estratos noemáticos y de noesis específicamente afectivas que permiten dar cuenta de un objeto con múltiples dimensiones de sentido y ya no de un mero objeto teórico. El punto de vista fenomenológico trascendental incorporó al esquema de las Investigaciones Lógicas una intencionalidad verdaderamente valorativa. Sin embargo, la exigencia de que los valores sean dados en actos específicamente emotivos choca con una

49 Recordemos la citada afirmación de Husserl (Supra, p. 13). Sobre estos límites de la primera ética de Husserl véase también Vargas Bejarano (2007); Peucker (2008). 
concepción de la intencionalidad y la constitución estrechamente asociada en este periodo a la idea de referencia objetiva. Hemos visto que Husserl busca una solución a este problema en un modelo de colaboración entre razón teórica y afectiva. Sin embargo, a la vez que contribuye a garantizar la racionalidad de los actos afectivos, este modelo introduce caracteres dóxicos en el acto de valoración que arrojan ciertas sospechas sobre la autonomía de la esfera afectiva. Cabe preguntarse entonces si es realmente posible concebir una verdadera intencionalidad valorativa sin sacrificar su carácter específicamente afectivo.

Si la respuesta ha de ser afirmativa, habrá que esperar aún algunos años para que la fenomenología husserliana cuente con los elementos que lo justifiquen. Para ser más precisos, es la ampliación de la intencionalidad hacia el ámbito de la experiencia pasiva, gracias a la elaboración del método genético, lo que permite a Husserl dar cuenta de una constitución afectiva totalmente desligada de una toma de posición activa sobre el objeto. Más aún, en el retroceso al nivel pre-yoico, el nivel más elemental de la vida de conciencia, Husserl podrá referirse a un pre-delineamiento de la constitución del mundo en el que el sentimiento tiene un rol incluso en el nivel de la configuración de los datos de sensación. Si bien lo que afecta al yo continua siendo ajeno al él, lo novedoso aquí es que el dato sólo puede afectar en tanto el yo se siente atraído, de modo tal que se vuelve hacia él confiriéndole cierto "valor", a fin de convertirlo en aparición de un objeto. En este nivel retrospectivo al que Husserl se refiere en los manuscritos del grupo $\mathrm{C}^{50}$ se desvela una estructura originaria (Urstruktur) en la que el sentimiento en relación al agrado o desagrado que provoca el dato tiene un lugar constitutivo junto a las kinestesias y los datos hyléticos. Aquí ya no es preciso hablar de una intencionalidad en el sentido de referencia objetiva y de lo valorado como un estrato fundado, sino de una intencionalidad de carácter instintivo y de cualidades valiosas verdaderamente pre-objetivas.

No obstante, al mismo tiempo que logra dar cuenta de una constitución afectiva carente de todo contenido dóxico, el problema inicialmente planteado pierde, para Husserl, su sentido. Esto es así en tanto cada vez más la distinción tajante entre tipos de actos será considerada abstracta a partir de la idea de que es imposible distinguir definitivamente la captación teórica de un objeto de su valoración. Por su parte, la fundamentación unilateral de los actos emocionales en los actos dóxicos será matizada en favor de la idea de que todos los tipos de actos están sujetos a una fundamentación mutua. Correlativamente, Husserl comenzará a elaborar una concepción de la razón que ya no admite ninguna diferenciación tajante entre sus distintos ámbitos y que desplaza la "soberanía universal" desde la dimensión lógica hacia la ética como doctrina universal de la razón (Vernunftlehre). En sintonía con esto, la segunda fase de su pensamiento ético restringirá el estudio de los actos y sus correlatos para enriquecer el punto de vista sobre la subjetividad y, si bien el víncu-

50 Husserl (2006), p. 318 ss. (Ms C 16, No 69). 
lo entre ética y razón no será abandonado, él será considerablemente profundizado a partir del establecimiento de una relación cada vez más estrecha entre razón y valores personales. A partir de este vínculo, Husserl emprenderá un tránsito hacia una concepción personalista de la ética fundada en valores provenientes del sentimiento. Gran parte de los cambios a los que nos referimos se relacionan con la influencia que la ética de M. Scheler ha ejercido sobre su maestro. Una explicación completa de las dimensiones de esta influencia excedería el tema del que aquí nos ocupamos pues atañe a problemas éticos que van más allá de la investigación descriptiva de los actos. De modo breve podemos decir que indudablemente ha sido Scheler quien sostuvo con mayor énfasis que la captación del valor prescinde de la mediación de actos representativos y que es ella la que permite la individualización de los objetos de conocimiento. Más aún, a esta primacía del valor del lado objetivo corresponde, de acuerdo con Scheler, la primacía de los actos emotivos y entre ellos, de una esfera de actos, que luego Husserl considerará como principio clave de individuación personal: los actos de amor ${ }^{51}$. En resumen, probablemente como consecuencia de algunos problemas a los que nos hemos referido e indudablemente gracias al creciente interés por sacar de su anonimato al sujeto de los actos de los que aquí nos hemos ocupado, muy distintos serán los temas que Husserl considerará importantes para la elaboración de una ética a partir de los años 20.

\section{Referencias bibliográficas}

FERRER, U. (1995): "De la teoría general del valor a una ética de fines, en Husserl", Anuario Filosófico, 28, pp. 41-60.

FERRER, U. (2012): “Razón axiológica y práctica en Husserl”, Veritas, 27, pp. 7795.

Ferrer, U; SÁnchez, S. (2011): La ética de Edmund Husserl, Madrid/Sevilla, Thémata- Plaza y Valdés.

Husserl, E. (2009): Investigaciones Lógicas, Manuel García Morente y José Gaos (trads.) Madrid, Alianza Editorial.

HUSSERL, E. (1985): Ideas relativas a una fenomenología pura y una filosofía fenomenológica. Libro Primero: Introducción general a la fenomenología pura. José Gaos (trad.) México, Fondo de Cultura Económica

HUSSERL, E. (2005): Ideas relativas a una fenomenología pura y una filosofía fenomenológica. Libro Segundo: Investigaciones fenomenológicas sobre la constitución. Antonio Zirión Q. (trad.) México, Fondo de Cultura Económica.

HuSSERL, E. (1988): Vorlesungen über Ethik und Wertlehre 1908-1914, Husserliana XXVIII, Dordrecht/ Boston/London, Kluwer Academic Publishers.

51 Cf. Scheler (1996). 
HusSerl, E. (2004): Einleitung in die Ethik. Vorlesungen Sommersemester 1920/1924, Husserliana XXXVII. Dordrecht/Boston/London, Kluwer Academic Publishers.

Husserl, E. (2006): Späte Texte über Zeitkonstitution (1929-1934): Die CManuskripte, Husserliana M. VIII, Dordrecht, Springer, 2006.

IrIBARNE, J. (2007): De la ética a la metafisica. En la perspectiva del pensamiento de Edmund Husserl. Bogotá, Universidad Pedagógica Nacional.

Melle, U. (1988): "Einleitung des Herausgebers", en U. Melle (ed.), Vorlesungen über Ethik und Wertlehre 1908-1914, Dordrecht/Boston/London, Kluwer Academic Publishers, pp. 13-49.

Melle, U. (1990): "Objektivierende und nicht-objektivierende Akte", en Samuel Ijsseling (ed.) Husserls-Ausgabe und Husserls-Forschung, Phaenomenologica 115, Dordrecht/Boston/ London, Kluwer Academic Publishers, pp. 35-49.

Melle, U. (1997): "Husserl's Phenomenology of Willing", en J.G.Hart y L.Embree (eds.), Phenomenology of Values and Valuing. Dordrecht/Boston/London, Kluwer Academic Publishers.

Melle, U. (2002): "Edmund Husserl: From Reason to Love", en J. J. Drummond y L. Embree (eds.) Phenomenological Approaches to Moral Philosophy, Dordrecht/Boston/London, Kluwer Academic Publishers.

Melle, U. (2012): "Husserls deskriptive Erforschung der Gefühlserlebnisse", en R. Breeur y U. Melle (eds.), Life, Subjectivity \& Art: Essays in Honor of Rudolf Bernet, Phaenomenologica 201. Dordrecht, Springer.

Peucker, H. (2008): "From logic to the person: an introduction to Edmund Husserl's ethics", The Review of Methaphysics, 62, pp. 307-325.

Rотн, A. (1960): Edmund Husserls ethische Untersuchungen, La Haya, Martinus Nijhoff.

SERrano de Haro, A. (1995): "Actos básicos y actos fundados. Exposición crítica de los primeros análisis husserlianos", Anuario Filosófico, 28, pp. 61-89.

SCHEler, M. (1996) Ordo Amoris, Madrid, Caparros Editores.

Schuhmann, K. (1991): "Probleme der Husserlschen Wertlehre", en Philosophisches Jahrbuch, 98, pp. 106-113.

VARgas Bejarano, J.C (2007): "La ética fenomenológica de Edmund Husserl como ética de la 'Renovación' y ética personal”, en Estudios de Filosofía, 36, pp. 61-93.

VicuÑa Zauschkevich, E. (2010): Para una fenomenología de la acción, aproximación a una teoría husserliana de la acción a partir de la idea de cumplimiento (Erfüllung) práctico. Pamplona, Cuadernos de Anuario Filosófico.

Celia Cabrera

Universidad de Buenos Aires / CONICET, Argentina

celi.filo@gmail.com 